\title{
To B or not to B: Arsenophonus as a source of B-vitamins in whiteflies.
}

\author{
Diego Santos-Garcia ${ }^{1, \dagger, *}$, Ksenia Juravel ${ }^{1}$, Shiri Freilich ${ }^{2}$, Einat Zchori-Fein ${ }^{3}$, \\ Amparo Latorre ${ }^{4,5}$, Andrés Moya ${ }^{4,5}$, Shai Morin ${ }^{1}$ and Francisco J. Silva ${ }^{4,5, \dagger, *}$ \\ 1 Department of Entomology, The Hebrew University of Jerusalem, Rehovot, Israel \\ 2 Institute of Plant Sciences, Newe-Ya'ar Research Center, ARO, Ramat-Yishai, Israel \\ 3 Department of Entomology, Newe-Ya'ar Research Center, ARO, Ramat-Yishai, Israel \\ ${ }^{4}$ Institute for Integrative Systems Biology (I2SysBio), Universitat de València-CSIC, Spain \\ ${ }^{5}$ Unidad Mixta de Investigación en Genómica y Salud (FISABIO/Universitat de València-I2SysBio), València, Spain \\ $\dagger$ Equal contribution. \\ * For correspondence: diego.santos@mail.huji.ac.il and francisco.silva@uv.es
}

\section{Abstract}

Insect lineages feeding on nutritionally restricted diets such as phloem, xylem, or blood, were able to diversify by acquiring bacterial species that complemented the missing nutrients. These bacteria, considered obligate/primary endosymbionts, share a long evolutionary history with their hosts. In some cases, however, these endosymbionts are not able to fulfill all the nutritional requirements of their host, driving the acquisition of additional symbiotic species. Whiteflies, which feed on phloem, established an obligate relationship with Candidatus Portiera aleyrodidarum, who provides essential amino acids and carotenoids to the host. As many Whiteflies species harbor additional endosymbionts, they could provide their hosts with missing nutrients. To test this hypothesis, genomes of several endosymbionts from the whiteflies Aleurodicus dispersus, A. floccissimus and Trialeurodes vaporariorum were sequenced and analyzed. All three species were found to harbor an endosymbiont from the genus Arsenophonus, and the two former also host Wolbachia. A comparative analysis of the three Arsenophonus genomes revealed that although all of them are capable of synthesizing B-vitamins and cofactors, such as pyridoxal, riboflavin, or folate, their genomes and phylogenetic relationship vary greatly. Arsenophonus of A. floccissimus and T. vaporariorum belong to the same clade and display characteristics of facultative endosymbionts, such as large genomes $(3 \mathrm{Mb})$ with thousands of genes, many pseudogenes, intermediate GC content, and mobile genetic elements (MGEs). In contrast, Arsenophonus of A. dispersus belongs to a different lineage and displays the characteristics of a primary endosymbiont, such as a reduced genome $(670 \mathrm{~kb})$ with 400 genes, $32 \%$ GC content, and no MGEs. However, the presence of 274 pseudogenes suggests that this symbiotic association is more recent than other reported hemipteran's primary endosymbionts. Arsenophonus of A. dispersus gene repertoire is completely integrated in the symbiotic consortia, and the biosynthesis of most vitamins occurs in shared pathways with its host. In addition, Wolbachia have also retained the ability to produce riboflavin, FAD, and folate, and may have a nutritional contribution. Taken together, our results show that Arsenophonus have a pivotal place in whiteflies nutrition by their ability to produce B-vitamins, even if they diverge and/or go through a genome reduction process.

Keywords: whitefly, symbiosis, vitamins, genome reduction, Arsenophonus, Wolbachia, metabolic complementation, riboflavin 


\section{Introduction}

Diverse eukaryotes live together with bacterial symbionts. Mutualistic, commensal, or parasitic relationships have been described for these associations (Moya et al., 2008). The Class Insecta is an example of mutualistic associations and several lineages are known to live in intimate relationship with an obligate symbiont for millions of years. Such symbionts are usually harbored inside specialized cells, termed bacteriocytes, and are vertically transmitted from the mother to her offspring. These bacterial symbionts, living inside cells and transmitted for long evolutionary periods, has been denominated primary (or obligatory) endosymbionts. The evolution of these symbioses had several effects on the gene repertoires of both hosts and endosymbionts, but is most notedly characterized by drastic reductions in the latter (Latorre and Manzano-Marín, 2016). Host diet complementation has frequently initiated the symbiosis, but in some cases, the host has nutritional requirements which the primary endosymbiont is unable to satisfy, and secondary (or facultative) endosymbiont are thus required for diet complementation. When these requirements became vital, it is possible that a secondary endosymbiont becomes primary, resulting in a consortium of primary endosymbionts.

Whiteflies and psyllids are two sister hemipteran lineages, which probably started their evolutionary success through the ancient association with ancestral bacterial species of the family Halomonadaceae (Santos-Garcia et al., 2014a). This bacterial lineage splits into Candidatus Portiera aleyrodidarum (hereafter, the term Candidatus will be used only at the first time a species is mentioned) in whiteflies and Candidatus Carsonella ruddii in psyllids. Based on gene repertoires, it was proposed that Portiera collaborates with its hosts in the synthesis of amino acids and carotenoids (Santos-Garcia et al., 2012, 2015; Sloan and Moran, 2012). Because whiteflies feed only on the phloem sieve-element, their diet is also deficient in vitamins and cofactors. Individual whiteflies may carry one or several additional endosymbionts belonging to seven genera (Zchori-Fein et al., 2014). It has been proposed that some of these additional endosymbionts are likely involved in vitamins and cofactors supply to their host, as for example, Candidatus Hamiltonella defensa (Rao et al., 2015). Candidatus Arsenophonus is a genus of secondary endosymbiont that has been observed in many whitefly species (Thao and Baumann, 2004, Zchori-Fein et al., 2014, Santos-Garcia et al., 2015). Similar to Hamiltonella, it is only found inside bacteriocytes, while other endosymbionts like Candidatus Wolbachia sp. or Candiddatus Rickettsia sp. present different tissue tropisms Gottlieb et al., 2008; Skaljac et al., 2010, 2013, Marubayashi et al., 2014). Arsenophonus lineages have also evolved intimate associations with other insect taxa with different diets, such as blood-sucking insects, where the supplementation of B-vitamins and cofactors were their proposed role (Šochová et al., 2017).

Whiteflies (Sternorrhyncha:Aleyrodidae) are composed mainly by two subfamilies, the Aleurodicinae and the Aleyrodinae. These two subfamilies probably originated between the Jurassic (Drohojowska and Szwedo, 2015; Shcherbakov, 2000) and the Middle Cretaceous (Campbell et al., 1994). While the Aleyrodinae contains the largest number of species described so far (140 genera, 1440 species), the Aleurodicinae has a relatively low number of described species (17 genera, 120 species) (Ouvrard and Martin, 2018). Analysis of the genome content of Portiera from the whitefly species Aleurodicus dispersus and A. floccissimus (Aleurodicinae) and Trialeurodes vaporariorum (Aleyrodinae) suggested that they are unable to complement their host diet with essential cofactors and vitamins (Santos-Garcia et al., 2015). The work presented here was thus initiated in order to test the prediction that Arsenophonus and/or Wolbachia, the other symbionts present in these species, are capable of providing their hosts with the lacking cofactors and vitamins. 


\section{Material and Methods}

\section{Sequences retrieval}

Sequences of bacteria other than Portiera were retrieved from a previous shotgun sequencing project of three whiteflies species: A. dispersus, A. floccissimus, and T. vaporariorum (SantosGarcia et al., 2015). In brief, T. vaporariorum was collected in 2014 near the IRTA Institute of Agrifood Research and Technology (Barcelona, Spain) and identified by Dr. Francisco José Beitia. A. dispersus and A. floccissimus were collected and identified by Dr. Estrella Hernandez Suarez in 2014 from banana fields (Tenerife Island, Spain). All three whiteflies harbored Portiera, Arsenophonus and Wolbachia endosymbionts. Genomic DNA (gDNA) was obtained using and alkaline lysis method from single bacteriomes (dissected with glass micro-needles). For each species, ten single bacteriome gDNA extractions were subjected to a Whole Genome Amplification process (WGA; GenomiPhi V2, GE Healthcare) and pooled by species. WGA gDNAs were sequenced by Illumina HiSeq 2000 using a mate-paired library $(100 \mathrm{bp} * 2$, 3kb insert size). A full description can be found at Santos-Garcia et al. (2015).

\section{Metagenome-assembled genomes (MAGs)}

For each species library, RAW Illumina reads were quality checked, and trimmed/clipped if necessary, with FastQC v0.11.3 (Andrews, 2010) and TrimmomaticPE v0.33 (Bolger et al., 2014). Kraken v0.10.6 (Wood and Salzberg, 2014) was used to classify the RAW Illumina reads using a custom genomic database including: Portiera, Hamiltonella, Rickettsia, Wolbachia, Arsenophonus, Candidatus Cardinium hertigii, several whiteflies' mitochondria, Bemisia tabaci MEAM1, and Acyrthosiphon pisum (Table S1). Reads classified as mitochondrial, insect or Portiera were discarded. The rest of reads were assembled with SPADES v3.11.0 (-meta -careful -mp) (Nurk et al., 2017). Obtained contigs were classified using Kraken and the custom database. Contigs belonging to known whiteflies endosymbionts were recovered and added to the custom database. Trimmed/clipped reads were re-classified and reads belonging to known whitefly endosymbionts were re-assembled alone with SPADES (-careful - mp) to produce metagenomeassembled genomes (MAGs). Illumina reads were digitally normalized (khmer v1.1) before the re-assembly stage (Crusoe et al., 2015). MAGs contigs were scaffolded and gap-filled with SSPACE v3 (Boetzer et al., 2011) and Gapfiller v1.10 (Boetzer and Pirovano, 2012), respectively. Finally, a manual iterative mapping approach, using Illumina trimmed/clipped reads, was performed with Bowtie2 v2.2.6 (Langmead and Salzberg, 2012), MIRA v4.9.5 (mapping mode) (Chevreux, B., Wetter, T. and Suhai, 1999), and Gap4 (Staden et al., 2000) until no more contigs/reads were joined/recovered for each MAG. Finally, Bowtie2 and Pilon v.1.21 (-jumps) (Walker et al., 2014) with Illumina reads were used to correct MAGs contigs.

\section{MAGs annotation}

MAGs initial annotations were performed with prokka v1.12 (Seemann, 2014). Enzyme commission numbers were added with PRIAM March_2015 release (Claudel-Renard et al., 2003). Gene Ontology, PFAM, and InterPro terms were added with InterProScan v5.27-66 (Jones et al., 2014). Putative pseudogenes and their positions in the genome were detected with LAST using several bacterium relative proteomes as a query (Kielbasa et al., 2011). Insertion Sequences (IS) were predicted with ISsaga (Varani et al., 2011). B. tabaci genome annotations (GCA_001854935.1) were downloaded and refined with PRIAM and InterProScan. MAGs, their corresponding Portiera (Santos-Garcia et al., 2015) and B. tabaci metabolism comparisons were 
performed on PathwayTools v21.5 (Karp et al., 2002). Heatmap and clustering was performed on R (R Core Team, 2018) with ggplots2 (Wickham, 2009).

\section{Comparative genomics}

OrthoMCL v2.0.9 was used to compute clusters of orthologous proteins (Li et al., 2003). Cluster of Orthologous Groups (COGs) terms were assigned using diamond v0.8.11.73 (July 2016 bacterial RefSeq database, Buchfink et al. (2015)) and MEGAN6 (Huson et al., 2016). Average Nucleotide Identity (ANI) and Average Amino Acid Identity (AAI) values were obtained with the Enveomics tools (Rodriguez-R and Konstantinidis, 2016). Synteny between MAGs was checked with Mummer v3 (Kurtz et al., 2004) and Mauve, using Arsenophonus ARAD as reference for contig re-ordering (Darling et al., 2010). GenoPlotR was used to plot Mauve results (Guy et al., 2010).

The presence of potential orthologs of B. tabaci genes in the other whiteflies species was assessed using the Illumina cleaned data and diamond, with a blastx search strategy (minimum alignment length of $25 \mathrm{bp}$ and $1 e^{-3}$ e-value) against the selected $B$. tabaci proteins (Table S2). Recovered reads were classified with Kraken, using the custom and the mini-kraken database, to discard bacterial reads. Finally, non-bacterial reads were used as query for a blastx search against the nr database (last access: March 2018) (Altschul et al., 1990) and their best hit classified with MEGAN6. Only reads assigned to the phylum "Arthropoda" were considered as genomic reads of the screened genes in the other whiteflies species.

\section{Phylogenetics}

General phylogenetic position of the newly sequenced Arsenophonus and Wolbachia was assessed using several 16S rRNA genes downloaded from the GenBank. Genes were aligned with ssu-align (default masking) (Nawrocki, 2009). IQ-TREE was used to select the best substitution model ( TVM+F+R3 and $\mathrm{TN}+\mathrm{F}+\mathrm{R} 2$, respectively) and to infer the majority rule maximum likelihood (ML) tree, with its associated support values (Nguyen et al., 2015; Kalyaanamoorthy et al., 2017).

Phylogenomic trees were generated using 82 and 97 conserved proteins, selected using PhyloPhlAn v0.99 (Segata et al., 2013), from Arsenophonus and Wolbachia inferred proteomes, respectively. Protein files were sorted by species name, using fastasort from exonerate v2.2.0 (Slater and Birney, 2005) and aligned with MAFFT v7.215 (Katoh et al., 2002). Proteins alignments were concatenated by species index using Geneious version 11.1.2 (Kearse et al., 2012). IQ-TREE was used to infer majority rule ML tree, and associated support values, under the substitution models JTTDCMut $+\mathrm{F}+\mathrm{R} 3$ for Arsenophonus and JTT $+\mathrm{F}+\mathrm{I}+\mathrm{G} 4$ for Wolbachia.

\section{Results}

\section{Genomic features and comparative genomics}

Five MAGs, two Arsenophonus and two Wolbachia from A. dispersus (ARAD, WBAD) and A. floccissimus (ARAF, WBAF) and one Arsenophonus from T. vaporariorum (ARTV) were recovered (Table 1). All MAGs were in a draft status but with different quality. Only ARAD, was assembled as a circular scaffold, supported by some mate-paired reads. However, a gap at the contig edges was not closed due to the presence of a dnaX duplication and a long AT low complexity region. The assembly of ARAF genome was also of high quality, with only 11 
contigs and a N50 value higher than $500 \mathrm{~kb}$. The rest of recovered MAGs were in different draft status, being the Wolbachia the most fragmented ones. Although Wolbachia was previously detected by PCR in T. vaporariorum (Santos-Garcia et al., 2015), its genome was impossible to assemble and analyze due to the low amount of reads recovered (Table S3). Indeed, only 21 Wolbachia contigs were recovered. From them, only one contig was above $1 \mathrm{~kb}$ (2.5X coverage) while the rest were below $400 \mathrm{nt}$. This suggests that Wolbachia is present in T. vaporariorum but at low amounts.

According to their genomic features, the Arsenophonus sequenced can be divided into two groups. While ARAD displays typical characteristics of primary endosymbionts, ARAF and ARTV display genomic features closer to facultative or secondary endosymbionts (Table 1). This pattern is emphasized by the different genome sizes ( 0.67 vs. $3 \mathrm{Mb})$, their GC content (32 vs. 37\%), the number of genes (428 vs. around 2000), and the presence of insertion sequences (ISs) and prophages ( 0 vs. 12). However, they have in common the low coding density (range 41 to 54\%) due to the presence of almost as many pseudogenes as genes. Most of the prophages in ARAF and ARTV were unrelated (based on their ANI values) except the pairs of prophages $2 / 8$ and $3 / 7$ from ARAF and the pair of prophage 9 from ARAF and prophage 7 from ARTV (Figure S1, Figure S2 and Table S4).

Regarding Wolbachia, both recovered MAGs display sizes and genomic features similar to other sequenced species from this genus (Ellegaard et al., 2013), with WBAF being more fragmented and lacking two tRNAs. Two incomplete prophages were detected in the genome of WBAD (Figure S1).

Table 1: Assembly statistics and genomic features of the five endosymbionts sequenced.

\begin{tabular}{|c|c|c|c|c|c|}
\hline & ARAD & ARAF & ARTV & WBAD & WBAF \\
\hline Species & Arsenophonus sp. & Arsenophonus sp. & Arsenophonus sp. & Wolbachia sp. & Wolbachia sp. \\
\hline Host & A. dispersus & A. floccissimus & T. vaporariorum & A. dispersus & A. floccissimus \\
\hline Genome size (bp) & 663125 & 3001875 & 3080136 & 1244696 & 1239495 \\
\hline Contigs & 1 & 11 & 93 & 237 & 422 \\
\hline N50 (bp) & - & 510728 & 236293 & 9433 & 5991 \\
\hline Coverage & 1698 & 2500 & 182 & 268 & 106 \\
\hline$\% \mathrm{GC}$ & 32 & 37 & 37 & 34 & 34 \\
\hline Genes & 428 & 1880 & 2312 & 990 & 909 \\
\hline CDS & 381 & 1781 & 2209 & 946 & 862 \\
\hline Noncoding RNA genes & 47 & 99 & 103 & 44 & 47 \\
\hline Coding density $^{\dagger}$ & 54 & 41 & 51 & 70 & 58 \\
\hline Avg. CDS length & 941 & 689 & 712 & 918 & 840 \\
\hline CDS \%GC & 36 & 39 & 39 & 34 & 34 \\
\hline $1 \mathrm{st}+2 \mathrm{nd} \% \mathrm{GC}$ & 42 & 42 & 42 & 38 & 38 \\
\hline $3 \mathrm{rd} \% \mathrm{GC}$ & 23 & 31 & 32 & 26 & 26 \\
\hline Pseudogenes & 274 & 1213 & 946 & 346 & 638 \\
\hline Avg. pseudo length & 461 & 1030 & 1011 & 586 & 527 \\
\hline Pseudo \%GC & 30 & 38 & 38 & 34 & 35 \\
\hline rRNA (16S|23S|5S) & $1|1| 3$ & $1|1| 4$ & $1|0| 9^{*}$ & $1|1| 1$ & $1|1| 1$ \\
\hline tRNA & 34 & 43 & 43 & 34 & 32 \\
\hline tmRNA & 1 & 1 & 1 & 1 & 1 \\
\hline other RNAs & 7 & 49 & 49 & 6 & 11 \\
\hline Insertion Sequences (IS) & 0 & 11 & $21^{*}$ & $5^{*}$ & $2^{*}$ \\
\hline Prophage regions & 0 & 12 & 12 & 2 & 0 \\
\hline Prophage regions size (bp) & - & 430067 & 336650 & 9948 & - \\
\hline Complete Prophage & - & 7 & 7 & 0 & - \\
\hline Incomplete Prophage & - & 5 & 5 & 2 & - \\
\hline
\end{tabular}




\section{Phylogenetic placement}

The phylogenetic position of each assembled Arsenophonus within the genus was tested using the $16 \mathrm{~S}$ rRNA phylogeny, phylogenomics and ANI/AAI analyses. Based on 16S rRNA phylogenetic analysis, ARAD belongs to a different lineage than ARAF and ARTV (Figure S3). While ARAD was placed in a not well supported cluster, including A. nasoniae and Arsenophonus from other insects (the aphid Stomaphis and the dipteran Ornithomya), ARAF and ARTV clustered with endosymbionts of several whitefly species and other insects. The use of a species threshold of 95\% ANI (Konstantinidis and Tiedje, 2005) suggests that A. triatominarum, $A$. nilaparvata, $A$. of Entylia carinata, ARAF and ARTV belong to the same species, whereas $A$. nasoniae, A. lipopteni and ARAD form three different species (Table S4, Figure S4). The AAI analysis showed the presence of two clusters, one for ARAD and A. nasoniae and the other for the remaining species, except the endosymbiont $A$. liptoteni, with a value smaller than $80 \%$ AAI. Phylogenomics results show a partial congruence with ANI/AAI, placing A. triatominarum as the closest relative of ARAD (Figure 1A). The two endosymbionts form a sister monophyletic clade to all the others Arsenophonus, which form a second monophyletic clade. A. nasoniae was placed at the basal position of both clades.

The phylogenetic positions of the two Wolbachia were tested with the same analyses. In the 16S rRNA phylogeny, WBAD (A. dispersus) and WBAF (A. floccissimus) form a well-supported clade inside a major clade, which contains Wolbachia strains from other whiteflies and other insect taxa (Figure S5). In addition, based on phylogenomics, WBAD and WBAF can be placed inside the Wolbachia B super group, with Wolbachia from Culex quinquefasciatus as the closest genome included in the analysis (Figure 1B). Finally, the high level of nucleotide identity (97.6\% ANI value) suggests that both Wolbachia, WBAD and WBAF, are strains from the same species. 

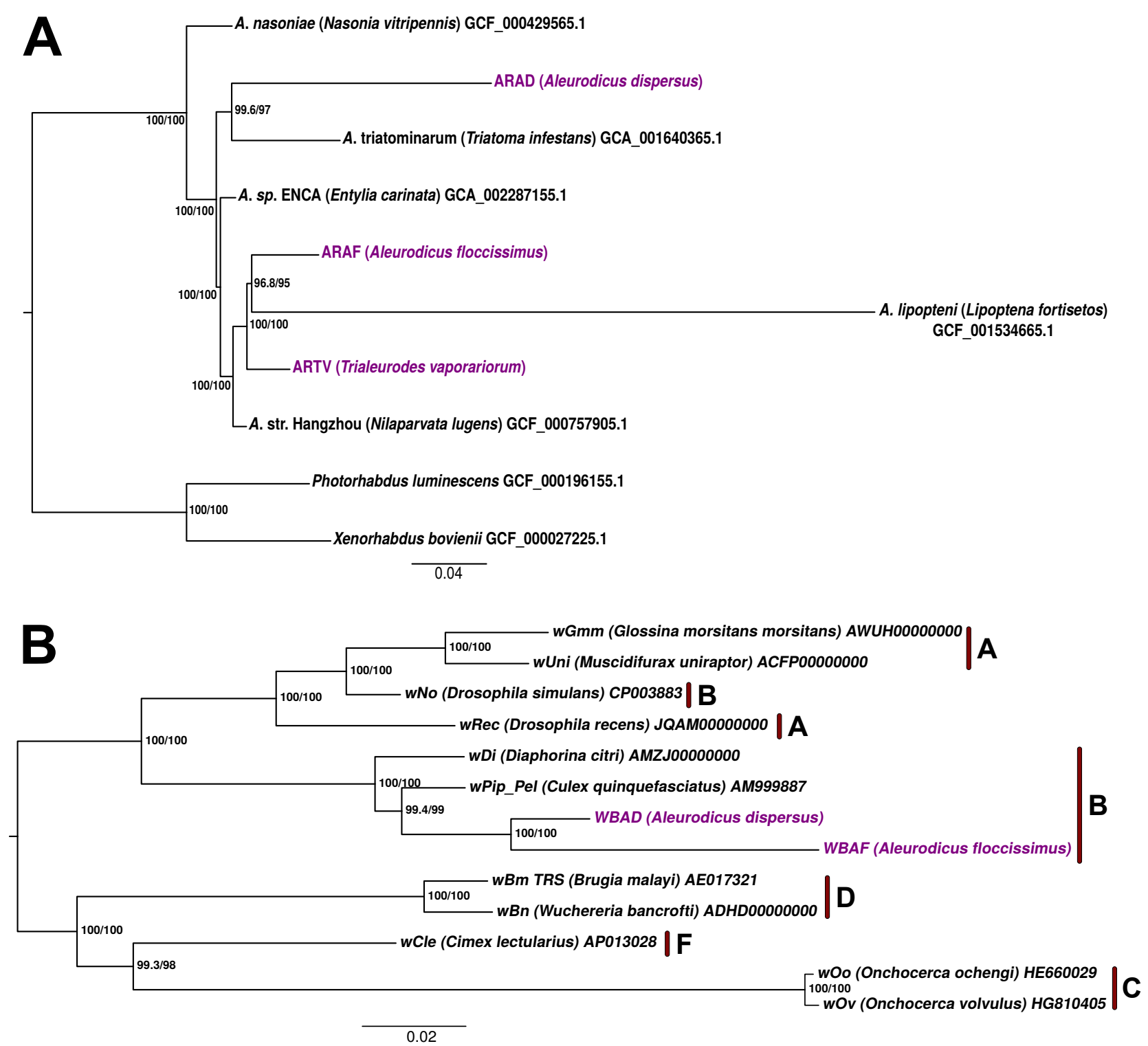

Figure 1: Phylogenomic trees of Arsenophonus and Wolbachia. (A) Arsenophonus (rooted) and (B) Wolbachia (unrooted) ML trees were inferred using 82 and 97 concatenated conserved protein alignments under a JTTDCMut $+\mathrm{F}+\mathrm{R} 3$ and JTT $+\mathrm{F}+\mathrm{I}+\mathrm{G} 4$ substitution model respectively. Support values were obtained with 1000 ultrafast bootstraps (right node labels) and 5000 SH-aLRT (left node labels). Names for the eukaryotic hosts are shown after the strain names inside parentheses. Accession numbers are displayed for each of the proteomes used. Arsenophonus ARAD, ARAF, and ARTV and Wolbachia WBAD and WBAF are highlighted in purple. (B) Red bars and letters denote the different Wolbachia supergroups. 
bioRxiv preprint doi: https://doi.org/10.1101/311076; this version posted June 28,2018 . The copyright holder for this preprint (which was not certified by peer review) is the author/funder, who has granted bioRxiv a license to display the preprint in perpetuity. It is made available under aCC-BY 4.0 International license.

\section{Comparative genomics: synteny and functional categories}

When the inferred proteomes from Arsenophonus ARAD, ARAF and ARTV were compared (Table S5), a core genome composed of only 289 clusters was obtained (Figure 2B). Interestingly, the core genome includes many clusters related to vitamins and cofactors biosynthetic pathways. The small number of shared protein clusters results from the reduced proteome of ARAD, which is mainly a subset of the larger ARTV and ARAF proteomes. Only 4 out of the 10 ARAD specific clusters were not hypothetical proteins: the pyruvate kinase II (pyruvate kinase I is present in the three proteomes), the 3-oxoacyl-[acyl-carrier-protein] synthase $(f a b F)$, which is involved in fatty acid and biotin biosynthesis, and proteins encoded by the genes $\operatorname{rec} A$ and nudE. In case only ARAF and ARTV were compared, the core proteome would have contained more than 1,000 clusters. The number of species specific clusters of ARTV (997) and ARAF (609) were higher compared to ARAD, which is in accordance with their genome sizes. Synteny analysis highlights the strong genome reduction underwent in ARAD compared to ARAF and ARTV (Figure 2A). This includes the loosing of macrosynteny (general genome architecture), while maintaining microsynteny (e.g. operons). In contrast, ARTV and ARAD still show high level of macrosynteny, although some rearrangements were observed (Figure 2C).

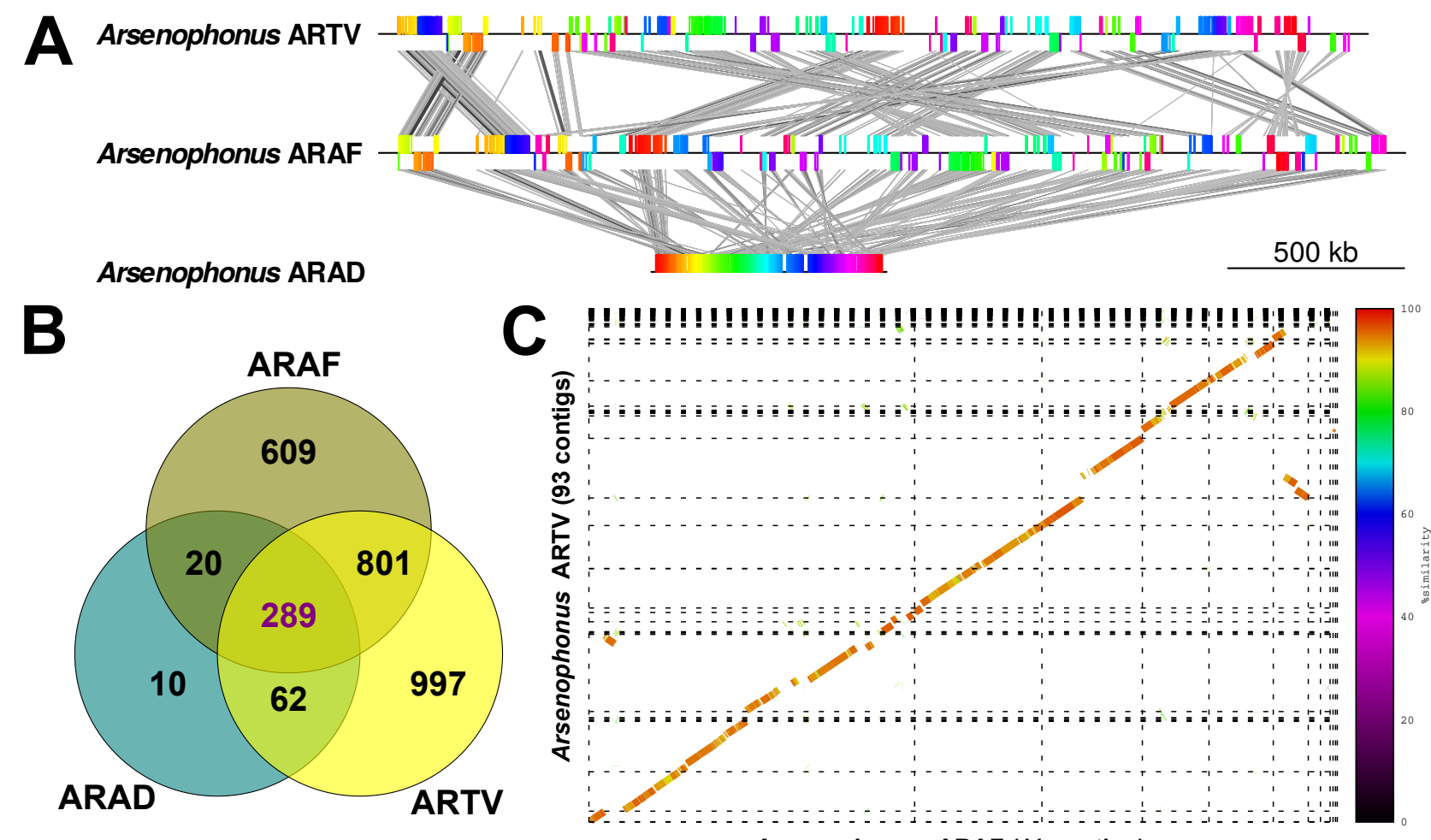

Arsenophonus ARAF (11 contigs)

Figure 2: Comparative analyses of the three Arsenophonus MAGs. (A) Graphic linear representation of the genome rearrangements observed comparing ARTV with ARAF and ARAF with ARAD. Synteny blocks display the same color. ARAF and ARTV contigs were ordered according to ARAD before synteny blocks calculations with Mauve for a better visualization. (B) Venn diagram displaying the number of clusters found on each subspace of the Arsenophonus pangenome (Table S5). (C) Synteny between the draft genomes of ARTV and ARAF. Contig edges are displayed as dashed lines. Contigs of ARTV were ordered with Mauve using ARAF as reference for a better visualization. 
specific clusters, respectively (Figure 3A, Table S6). It should be noted, however, that the highly fragmented status of these genomes could produce a large number of artificial specific clusters. Comparison of the two Wolbachia genomes revealed that some of the largest contigs show the same gene order and a high level of nucleotide identity (Figure 3B), suggesting that both macrosynteny and microsynteny are probably kept.

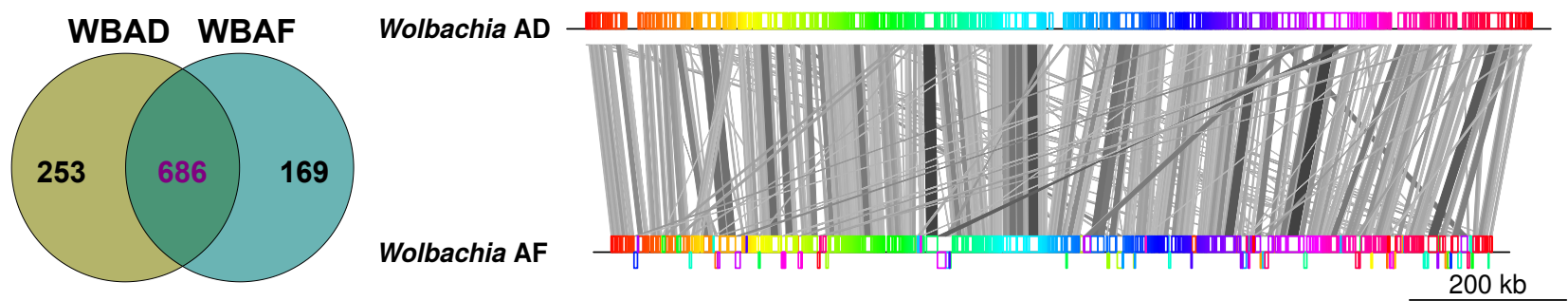

Figure 3: Comparative analyses of the two Wolbachia MAGs. (A) Venn diagram showing the shared and specific coding gene clusters between WBAD and WBAF (Table S6). (B) Synteny between the draft genomes (see Table 1) WBAD and WBAF. Contigs of WBAF were ordered with Mauve using WBAD as reference for a better visualization.

The proteomes of the five endosymbionts were functionally classified according to the Cluster of Orthologous Groups (COGs). Because of the strong reduction of the gene repertoire in Arsenophonus ARAD, this endosymbiont shows a smaller number of hits in each functional category, except J (translation and ribosomal structure and biogenesis), where the number of hits was similar to the other endosymbionts (Figure 4 left). In fact, in ARAD, this category has a relative frequency in the proteome higher than 0.204 right). In addition, category $\mathrm{J}$ and the other categories related to Information storage and processing $(\mathrm{A}, \mathrm{K}$, and $\mathrm{L}$ ) showed higher relative frequencies in ARAD than in the other two Arsenophonus (Figure 4 right). Based on the number of hits and relative frequencies, the loss of the gene repertoire in categories $\mathrm{G}$ (carbohydrate metabolism), I (lipid metabolism), and O (post-translational modification and chaperones) was lower in ARAD. As expected, Wolbachia proteomes show the retention of the informational categories but also retain COGs related, among others, to environmental response. 
bioRxiv preprint doi: https://doi.org/10.1101/311076; this version posted June 28, 2018. The copyright holder for this preprint (which was not certified by peer review) is the author/funder, who has granted bioRxiv a license to display the preprint in perpetuity. It is made available under aCC-BY 4.0 International license.

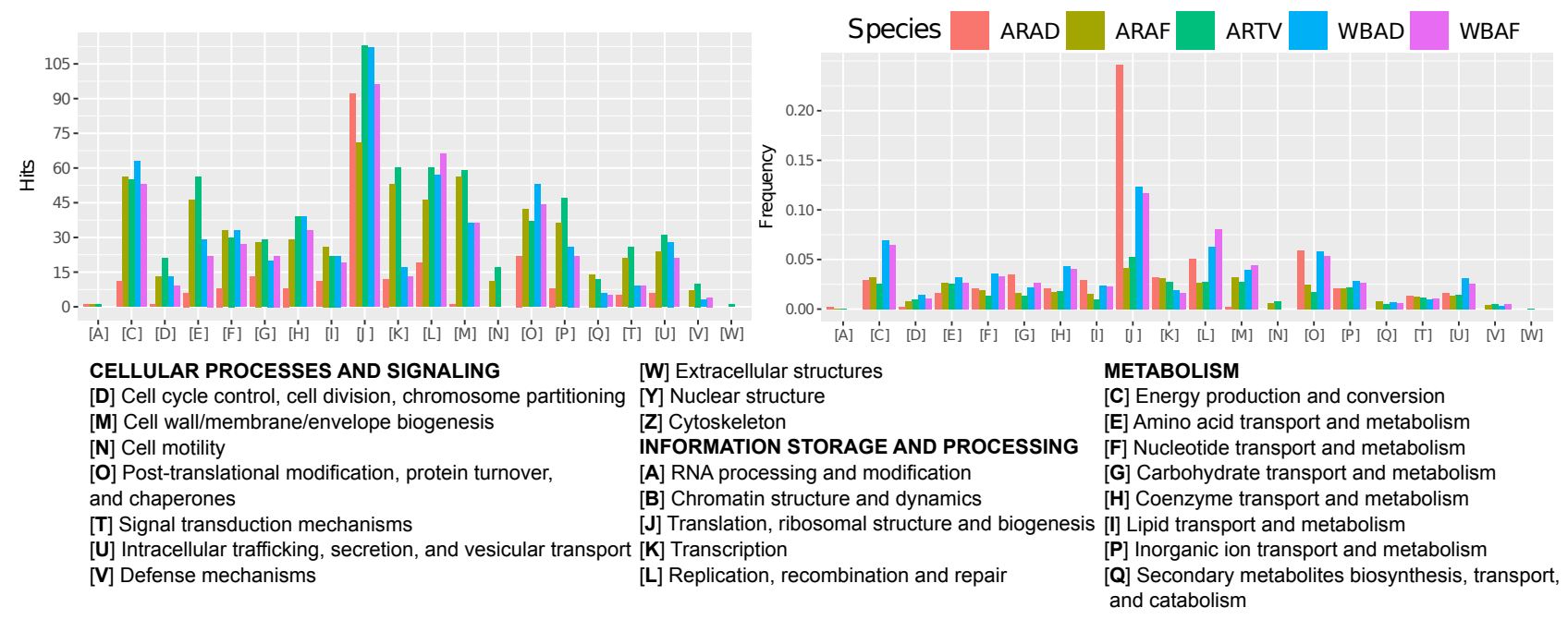

Figure 4: Distribution of the endosymbiont proteomes in functional categories. Bar plots showing the number of hits (left) and its relative frequency (right) in each functional category (COG) in the proteomes of the three analyzed Arsenophonus and the two analyzed Wolbachia MAGs. 


\section{Biosynthetic metabolic potential}

An integrated analysis of the metabolism of the whitefly bacteriocytes of the three species was performed in order to predict the potential biosynthetic capabilities of the harbored Arsenophonus, Wolbachia and Portiera (Santos-Garcia et al., 2015). That of the insect host was inferred using the B. tabaci genome (GCA_001854935.1). Amino acid biosynthesis in the three whiteflies is mainly conducted by the Portiera strains (PAAF, PAAD, and PATV), which maintain the ability to produce four (PATV)/five (PAAF and PAAD) out of ten essential amino acids, and require some complementation from the host for the synthesis of the others six (Figure 5 A, cluster A). While some cofactors/vitamins and precursors are produced by almost all the endosymbionts and the host (cluster B), others are mainly produced by some Arsenophonus (cluster C), both Arsenophonus ARAF and ARTV, Wolbachia and the host (cluster D), or, apparently, by none of them (cluster E). In general, both Wolbachia present a biosynthetic potential that is lower than that of the most reduced Arsenophonus, ARAD. In addition, while both Wolbachia still present all the electron transport chain, Arsenophonus ARAF and ARTV have lost the Ubiquinol oxidase and ARAD only maintains the Cytochrome-c oxidase and has lost the ATP synthase (not shown).

Although the essential amino acid biosynthetic potential of Arsenophonus and Wolbachia is limited (Figure 5A), ARAF and ARTV still retain some genes involved in the synthesis of amino acids or their precursors, such as lysine (its precursor, meso-diaminopimelate is also involved in the peptidoglycan biosynthesis) and chorismate (the precursor of phenylalanine). In ARTV, the complete pathway, encoded by seven genes, from D-erythrose 4-phosphate and D-ribulose-5-phosphate to chorismate is functional (aroF, aroB, aro $Q$, aroE, aroL, aro $A$, and $\operatorname{aro} C)$. This, in combination with the presence of $\operatorname{tyr} A, \operatorname{tgr} B$, and an additional monofunctional chorismate mutase gene, suggests the ability of ARTV to produce tyrosine and a potential collaboration with Portiera, or the host, for the production of phenylalanine. The ability to synthetize amino acid was completely lost in ARAD, suggesting this not to be the reason why the lineage of ARAD evolved a close association with $A$. dispersus.

The contribution of the different partners to the synthesis of vitamins and cofactors is heterogeneous. ARTV and ARAF, the two Arsenophonus with larger genomes, are those maintaining higher capabilities, with the loss of several genes required for some of the pathways. Despite the reductive evolutionary process in ARAD that has drastically reduced its potentialities, some capabilities are still retained, suggesting that they might be important in the symbiotic relation (Figure 5A and 5B). ARAD, but also ARAF and ARTV, encode the complete pathway to synthesize pyridoxal 5'-phosphate (vitamin B6) and riboflavin (vitamin B2). However, while ARAF and ARTV are able to produce FMN and FAD because they harbor a functional ribF gene, it is pseudogenized in ARAD. Since this gene is present in all whitefly hosts, both flavin cofactors may be produced in $A$. dispersus through a complementation between ARAD and its host (Figure 5B, Table S2). Also, being complemented again by the host, ARAD could synthesize thiamine (vitamin B1) and folate (vitamin B9, from imported chorismate) (Figure $5 \mathrm{~B})$. Although ARAD has lost thiH gene, some host encoded proteins can replace its function (Table S2). ARAF and ARTV also require complementation by their host in order to produce folate. ARTV lost most of the pathway to produce thiamine, and the missing steps are not complemented by its host. Additionally, B. tabaci seems to be able to produce biotin using some horizontally transferred genes (bioA, bioB, and bioD) (Luan et al., 2015), a protein capable of replacing the BioC function, and the insect's own fatty acid biosynthesis pathway. Genes with similar function to bioABCDFH genes were found to be present in $T$. vaporariorum genomic reads, suggesting that the whitefly is also capable of producing biotin by using only host genes. Because only genes similar to bioBCFH were identified in the genomic reads from both 
Aleurodicus species (Table S2), the presence of bioA and bioD in ARAD and ARAF is required for the cooperative synthesis of biotin. However, ARAD, unlike ARAF, has only retained the two missing genes in the genomes of both Aleurodicus.

In ARAF, the gene yigB which is part of the riboflavin pathway, is pseudogenized. This gene encodes for a Haloacid Dehalogenase (HAD) phosphatase, that belongs to a superfamily of enzymes. Therefore, the activity of the protein encoded by the gene, can be substituted by related genes in the endosymbiont or the host genomes (Manzano-Marín et al., 2015). Finally, all Arsenophonus conserve the glycolysis and the pentose phosphate pathways to produce the precursors required for riboflavin, thiamine, and pyridoxal. Indeed, in ARAD, the enzyme encoded by the gatA gene from the glycolysis pathway seems to have replaced the epd product in the pyridoxal pathway. The combination of $i l v C$ encoded enzyme in Portiera (Price and Wilson, 2014) and the pan $B C$ genes that were horizontally transferred to B. tabaci, allows this whitefly to produce its own pantothenate (vitamin B5) and Coenzyme A. However, panBC genes are likely not present in the genomes of $A$ dispersus, A. floccissimus, and T. vaporariorum, where only bacterial hits were recovered showing no similarity to the ones present in the $B$. tabaci genome. This suggests that these whiteflies are not able to produce pantothenate and they acquire it from the diet or other sources (Figure 5B and Table S2). However, failure on horizontal transferred genes detection could be an artifact from the methods used, since they are unable to discover new horizontally transferred genes in the whiteflies screened.

The third endosymbiont of $A$. dispersus and A. floccissimus, Wolbachia WBAD and WBAF, may be also contributing to the vitamin/cofactor synthesis of the system, as they have the complete pathway for riboflavin-FMN-FAD synthesis and potentially are able to produce folate from some intermediate precursors (Figure 5B). 


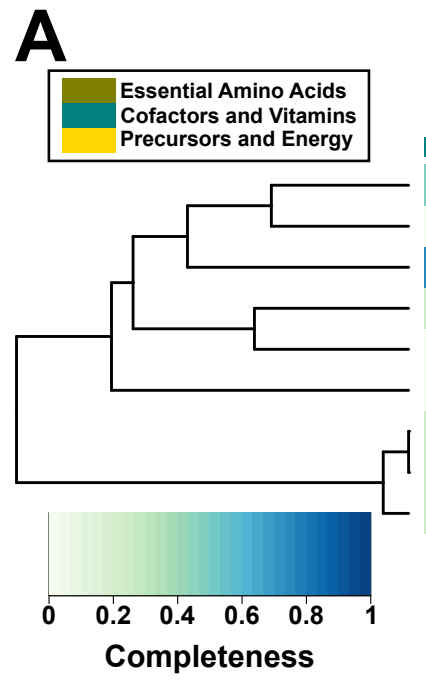

B
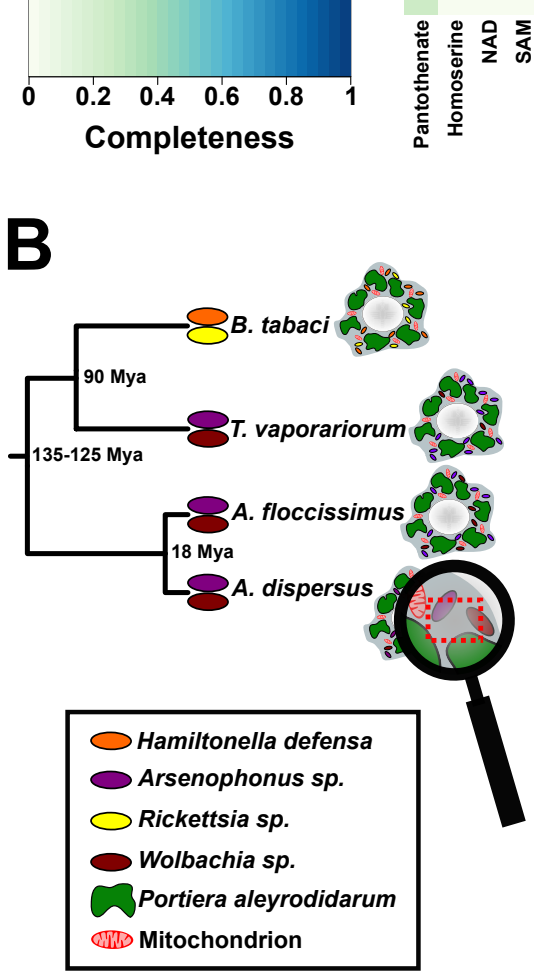

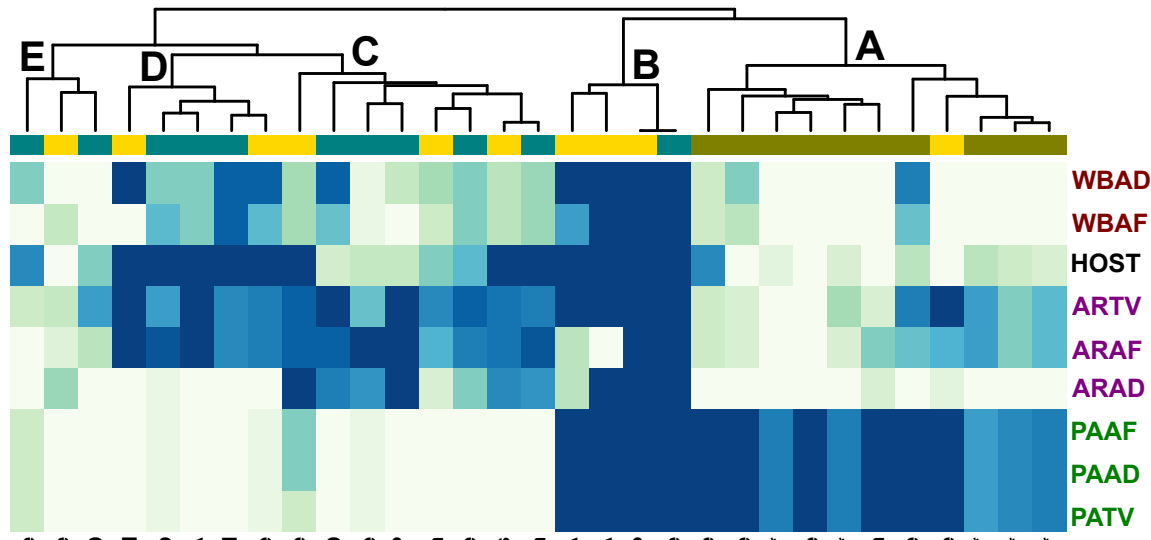

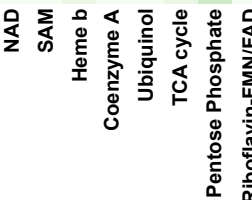

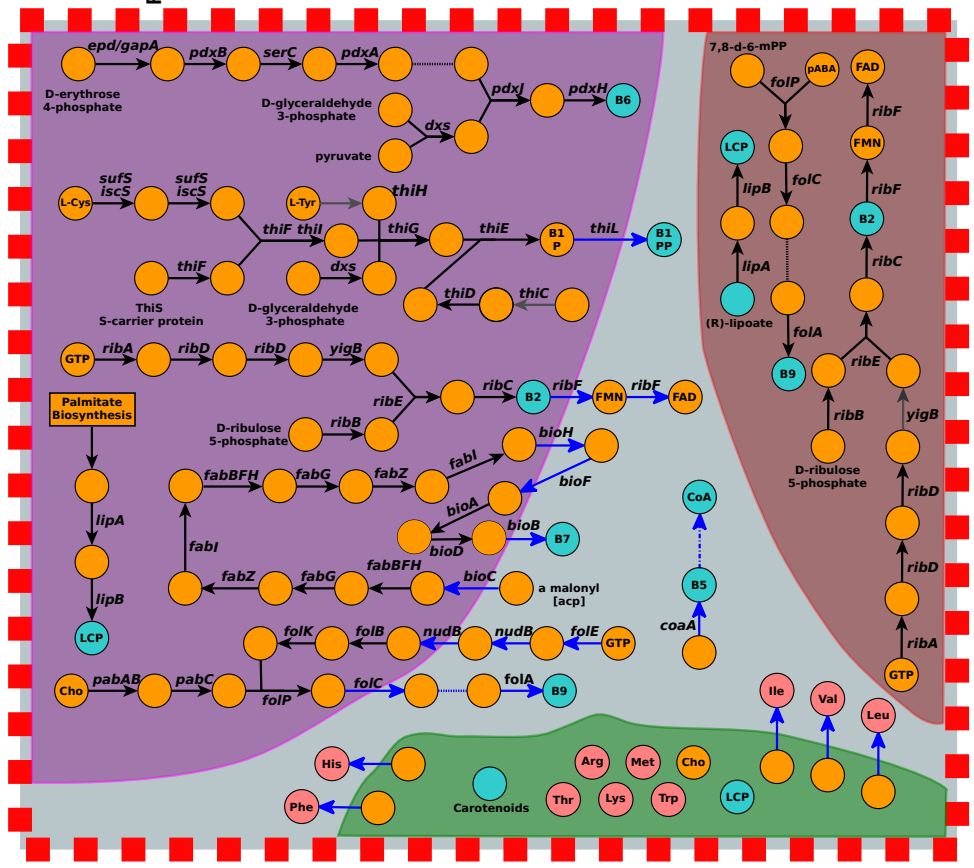

Figure 5: Biosynthetic potential of Arsenophonus, Wolbachia, Portiera and whiteflies. (A) Heat map and hierarchical clustering showing, in a color scale, the degrees of completeness of several compounds' biosynthetic pathways, including essential amino acids, cofactors and vitamins. Completeness was measured as number of enzymes present in the analyzed genome over the total number of enzymes in the pathway. Arsenophonus (AR), Wolbachia (WB) and Portiera (PA) from A. dispersus (AD), A. floccissimus (AF) and T. vaporariorum (TV) are highlighted in purple, red and green respectively. B. tabaci genome was used as a general host representative. (B) On the left, phylogenetic relationship and endosymbionts content of the Aleurodicus, T. vaporariorum and B. tabaci whiteflies (Santos-Garcia et al., 2015). Sequences from Wolbachia were scarcely detected in T. vaporariorum, suggesting low amounts of this bacterium in the insect. On the right, schematic representation of some vitamins and precursors metabolic pathways occurring at the bacteriocyte of A. dispersus. Arsenophonus ARAD is represented in purple, Wolbachia WBAD in redwood, Portiera in green and the host's cytosol in blue. Black arrows denote enzymes present in the corresponding endosymbiont, blue arrows enzymes present in the host, grey arrows denote pseudo/absent genes, dashed arrows denote simplified pathways and dashed lines spontaneous reactions. Abbreviations: lipoyl carrier protein (LCP), chorismate (Cho), acyl carrier protein (acp), 4-aminobenzoate (pABA), (7,8-dihydropterin-6-yl)methyl diphosphate (7,8zd-6-mPP), diphosphate (PP). 


\section{Discussion}

Some bacterial lineages show, predominantly, facultative symbiotic lifestyles associated with insects. In such cases, they can inhabit the cytoplasm of bacteriocytes and coexist with the primary endosymbiont. These bacterial lineages usually have large gene repertoires and genomes, allowing them to be autonomous and capable of infecting individuals from very different taxa. Examples of such clades are the genera Arsenophonus, Sodalis, and Serratia (Nováková et al. 2009; Manzano-Marín et al., 2017; Santos-Garcia et al., 2017). Because their presence may bare fitness costs to the host, they do not become fixed in the insect lineage except if their nutritional contribution (or other kind of benefit) is important and is required for periods of many generations. Under such a scenario, a facultative endosymbiont may evolve towards a more intimate association, becoming a co-primary symbiont (Lamelas et al., 2011). Although there are several features associated with this new lifestyle, the most relevant is the decrease in the genome size. In the lineage Arsenophonus-Riesia, the sizes of several genomes have been reported, ranging from the $0.58 \mathrm{Mb}$ of Candidatus Riesia pediculicola (Kirkness et al., 2010) and $0.84 \mathrm{Mb}$ of $A$. lipopteni (Nováková et al., 2016) to $3.86 \mathrm{Mb}$ of $A$. triatominarum, but with several genomes of intermediate sizes (Śochová et al., 2017). Similarly, Sodalis endosymbionts display also a large range of genome sizes, from the $0.35 \mathrm{Mb}$ of Candidatus Mikella endobia (Husnik and McCutcheon, 2016) to the circa $4.5 \mathrm{Mb}$ of S. glossinidius or S. pierantonius, and with several genome sizes in the range of 1-2 Mb (Santos-Garcia et al., 2017). This range has also been observed in Serratia symbiotica, with genome sizes from 0.65 to 3.86 Mb (Manzano-Marín et al., 2016).

Previous findings by Nováková et al. (2009) placed Arsenophonus from A. dispersus and $A$. duguesii (a close relative), together with an Arsenophonus from $B$. tabaci, in a clade with long branches, which is apart from other Arsenophonus of whiteflies. Our phylogenomics confirms these results, and have revealed that ARAD belongs to a different clade from the other two whiteflies' Arsenophonus, ARAF and ARTV. Two scenarios can explain the fact that ARAD and ARAF belong to different clades. In the first, the ancestor of ARAD infected the lineage of A. dispersus after its divergence from A. floccissimus and later started its shift to a primary symbiosis and its associated genome reduction process. In this case, the process could have taken place for as long as $18 \mathrm{My}$, the estimated divergence time between these two whitefly species (Santos-Garcia et al. 2015). An alternative scenario is that this association began prior to the divergence of the two Aleurodicus, but later, ARAD-type symbiont was replaced in the A. floccissimus lineage by another Arsenophonus lineage (ARAF). Although both scenarios are possible, the large number of pseudogenes in the ARAD genome suggests that the initiation of the process was relatively recent, as suggested by the first scenario. Still, the second scenario cannot be excluded as symbiont replacement has been documented in many insect lineages (Sudakaran et al., 2017).

As far as we know, Arsenophonus ARAD is among the smallest genomes in its genus, between R. pediculicola and A. lipopteni (Šochová et al., 2017). ARAD is still under a genome reduction process that could end up below $0.4 \mathrm{Mb}$ if the current $54 \%$ coding density is considered. ARAD gene repertoire is clearly a subset of other whiteflies' Arsenophonus. It is totally dependent on the host' environment, and putatively supplies/complements its host with cofactors/vitamins not produced by Portiera and the host. In conclusion, ARAD can be considered a co-primary endosymbiont. On the other hand, ARAF and ARTV are still at the beginning of the genome reductive process (e.g. high number of pseudogenes, inactivation of mobile genetic elements, and virulence/secretion factors). They are heritable facultative endosymbionts with the potential to establish a co-primary symbiotic relationship with Portiera and their host. However, as frequently observed, all Arsenophonus, independently of their symbiotic status, can be replaced 
by other bacterium of the same, or different genera, as long as the new comers have similar biosynthetic capabilities (Thao and Baumann, 2004; Russell et al., 2017; Sudakaran et al., 2017).

We hypothesize that the ability to synthesize riboflavin and other vitamins is probably the main reason for the presence of Arsenophonus endosymbionts in whiteflies and the evolution of ARAD towards a co-primary symbiont in A. dispersus. As indicated above, nutritionally poor diets such as blood and phloem sap, cannot provide insects with all their dietary requirements, including several vitamins. For example, the essentiality of dietary riboflavin was demonstrated in aposymbiotic aphids but not in aphids harboring Buchnera aphidicola, which is able to produce riboflavin (Nakabachi and Ishikawa, 1999). Moreover, B. aphidicola of aphids from the subfamily Lachninae already lost the genes encoding the pathway in the Lachninae ancestor. This led to the establishment in this group of a new association with an additional bacterium, capable of producing riboflavin. In most lineages, this is the co-primary S. symbiotica, a facultative endosymbiont in other aphid lineages (Manzano-Marín and Latorre, 2014). The analyses of the co-symbiosis in species of Lachninae revealed a complex system with S. symbiotica endosymbionts harboring a very small genome (in Tuberolachnus salignus), intermediate genome sizes (in some species of the genus Cinara), or replaced by other endosymbionts belonging to different genera, such as, Sodalis, Erwinia, or Hamiltonella, among others (Manzano-Marín et al., 2016, 2017; Meseguer et al., 2017). The essential role riboflavin biosynthesis might play in establishing symbiotic relationships has also been demonstrated in several blood-feeding arthropods harboring different endosymbionts, such as A. lipopteni and its host the biting fly Lipoptena cervi (Nováková et al., 2016), Wolbachia associated with the bedbug Cimex lectularius (Moriyama et al., 2015), and Coxiella-like bacteria found in several ticks (Gottlieb et al., 2015).

Although, thiamine, pantothenate, coenzyme A, niacin, pyridoxal and biotin can be found in the phloem (free or bounded to transporter proteins) (Pirson and Zimmermann, 1975), their concentrations might be not sufficient for many phloem-feeding species. This could explain why the capability to produce some of these compounds has been kept in bacterial endosymbionts while it has been lost in others. However, the production of one or several B vitamins (thiamine, riboflavin, pyridoxal, folate, and biotin) and de novo lipoate by whiteflies' Arsenophonus seems to be the main contribution of these endosymbionts to their hosts, allowing them to feed on the limited diet present in the phloem (Dale et al., 2006; Nováková et al., 2015, 2016; Mao et al. 2017). Regarding biotin biosynthesis, the bacterial origin of bioAB and bioD was reported by Luan et al. (2015) and Ankrah et al. (2017). We show here that not all whiteflies seem to have the horizontally transferred bioAD genes, although the rest of the bio operon homologs, including the bioB, are contained. This suggest that whiteflies acquired, by several horizontal gene transfer events, the ability to produce biotin. This is expected to produce a gradual loss of the biotin pathway in secondary endosymbionts of the different whiteflies lineages, at least from the Aleyrodinae subfamily, as this group harbors the full bio operon. Indeed, Cardinium hertigii infecting B. tabaci has recently lost its ability to produce biotin, raising the possibility that biotin is provided by the co-present Hamiltonella endosymbiont or by the host metabolic potential (Santos-Garcia et al., 2014b, Rao et al., 2015). As an alternative, this can also lead to intricate complementation patterns as shown in the Arsenophonus from Aleurodicus. It is important to notice that supplementation of vitamins was the role proposed for Hamiltonella in B. tabaci (Rao et al., 2015) and the results reported here support a similar function for Arsenophonus. Interestingly, these two endosymbionts are never found together in the same individual (Gottlieb et al., 2008; Skaljac et al., 2010, 2013; Marubayashi et al., 2014). It could be possible that the two endosymbionts might compete for the same host resources to produce the same compounds, undermining the unique benefit to the host if harbored together due to the cost associated of maintaining high endosymbionts loads (Ferrari and Vavre, 2011).

Finally, and in contrast to Arsenophonus, Wolbachia from Aleurodicus species only produces 
riboflavin and, probably, folate. It has been proven that the bedbug $C$. lectularius requires the riboflavin produced by Wolbachia for its proper development. Indeed, the riboflavin pathway is conserved among Wolbachia species infecting different insects, suggesting that it may provide a fitness benefit to the infected host (Moriyama et al., 2015). However, it is yet to be determined if Wolbachia can be considered as a general mutualistic symbiont (at the metabolic level) in whiteflies, like Hamiltonella or Arsenophonus. First, while Hamiltonella and Arsenophonus are restricted to bacteriocytes, Wolbachia is found in different tissues (Gottlieb et al., 2008; Skaljac et al., 2010, 2013; Marubayashi et al., 2014). While the first pattern is more characteristic of hemipteran mutualistic endosymbiosis, the second can be found in endosymbionts with a wide range of symbiotic interactions, including parasitism. Second, in most reported cases of whiteflies harboring Wolbachia, the individual insects usually harbor in addition Arsenophonus or Hamiltonella (Skaljac et al., 2010, 2013, Kapantaidaki et al., 2014, Marubayashi et al., 2014, Zchori-Fein et al. 2014). The few cases in which Wolbachia was found to be the unique secondary endosymbiont should be handled with caution, as they may simply result from failure of the general PCR primers to amplify other symbionts species/strains (Augustinos et al., 2011).

In conclusion, the loss of the genes encoding the enzymes for the synthesis of vitamins in the ancestral Portiera, many million years ago, likely generated the requirement of a co-symbiont in whiteflies. Arsenophonus species seem to be the most common co-symbiont. In the lineage of A. dispersus, the harbored Arsenophonus lineage presents a highly reduced genome content. This Arsenophonus lineage is, or is in the process of becoming, a co-primary symbiont which putatively supplies/complements its host with cofactors/vitamins that are not produced by its co-partner Portiera.

\section{Conflict of Interest Statement}

The authors declare that the research was conducted in the absence of any commercial or financial relationships that could be construed as a potential conflict of interest.

\section{Author Contributions}

DSG and FJS conceived the study. DSG and FJS performed bioinformatics analysis. KJ performed phylogenetics analysis. All authors analyzed and discussed the data. DSG and FJS drafted the manuscript with inputs from AL, AM, EZF, SF and SM. All authors participated in the revision of the manuscript.

\section{Funding}

This work was supported by the grants PROMETEOII/2014/065 (Conselleria d'Educació, Generalitat Valenciana, Spain) to AM, BFU2015-64322-C2-1-R (co-financed by FEDER funds and Ministerio de Economía y Competitividad, Spain) to AL, and by the ISRAEL SCIENCE FOUNDATION (Israel) grants No. 1039/12 to SM and No. 484/17 to SF and EZF.

\section{Acknowledgments}

DSG is recipient of a Golda Meir Post Doctoral Fellowship at the Hebrew University of Jerusalem. The authors acknowledge Francisco J. Beitia and Estrella Hernández Suárez for the whiteflies samples. 


\section{Supplemental Data}

The Supplementary Material for this article can be found online at: Supplementary Material

\section{Data Availability Statement}

The Arsenophonus (ERZ502704-6) and Wolbachia (ERZ502707-8) annotated genomes and the whole genome shotgun libraries (ERR2532344-45 and ERR2534068-71) analyzed for this study have been deposited at the European Nucleotide Archive (ENA) under the project number PRJEB26014. The generated Pathway Tools databases and the blastx results of the genomic reads similar to Bemisia tabaci key cofactors/vitamins metabolic genes can be found at $10.6084 / \mathrm{m} 9$. figshare.6142307.

\section{References}

Altschul, S. F., Gish, W., Miller, W., Myers, E. W., and Lipman, D. J. (1990). Basic local alignment search tool. J Mol Biol 215, 403-410. doi:10.1016/S0022-2836(05)80360-2

[Dataset] Andrews, S. (2010). Fastqc: A quality control tool for high throughput sequence data.

Ankrah, N. Y., Luan, J., and Douglas, A. E. (2017). Cooperative metabolism in a threepartner insect-bacterial symbiosis revealed by metabolic modeling. J Bacteriol 199, 1-15. doi:10.1128/JB.00872-16

Augustinos, A. A., Santos-Garcia, D., Dionyssopoulou, E., Moreira, M., Papapanagiotou, A., Scarvelakis, M., et al. (2011). Detection and characterization of Wolbachia infections in natural populations of aphids: is the hidden diversity fully unraveled? PLoS One 6, e28695. doi:10.1371/journal.pone.0028695

Boetzer, M., Henkel, C. V., Jansen, H. J., Butler, D., and Pirovano, W. (2011). Scaffolding pre-assembled contigs using SSPACE. Bioinformatics 27, 578-9. doi:10.1093/bioinformatics/ btq683

Boetzer, M. and Pirovano, W. (2012). Toward almost closed genomes with GapFiller. Genome Biol 13, R56. doi:10.1186/gb-2012-13-6-r56

Bolger, A. M., Lohse, M., and Usadel, B. (2014). Trimmomatic: a flexible trimmer for Illumina sequence data. Bioinformatics 30, 2114-2120. doi:10.1093/bioinformatics/btu170

Buchfink, B., Xie, C., and Huson, D. H. (2015). Fast and sensitive protein alignment using DIAMOND. Nat Methods 12, 59-60. doi:10.1038/nmeth.3176

Campbell, B. C., Steffen-Campbell, J. D., and Gill, R. J. (1994). Evolutionary origin of whiteflies (Hemiptera: Sternorrhyncha: Aleyrodidae) inferred from $18 \mathrm{~S}$ rDNA sequences. Insect Mol Biol 3, 73-88

Chevreux, B., Wetter, T. and Suhai, S. (1999). Genome Sequence Assembly Using Trace Signals and Additional Sequence Information. Computer Science and Biology: Proceedings of the German Conference on Bioinformatics (GCB) 99, 45-56

Claudel-Renard, C., Chevalet, C., Faraut, T., and Kahn, D. (2003). Enzyme-specific profiles for genome annotation: PRIAM. Nucleic Acids Res 31, 6633-6639. doi:10.1093/nar/gkg847 
Crusoe, M. R., Alameldin, H. F., Awad, S., Boucher, E., Caldwell, A., Cartwright, R., et al. (2015). The khmer software package: enabling efficient nucleotide sequence analysis. F1000Res 4, 900. doi:10.12688/f1000research.6924.1

Dale, C., Beeton, M., Harbison, C., Jones, T., Pontes, M., Al, D. E. T., et al. (2006). Isolation , Pure Culture, and Characterization of "Candidatus Arsenophonus arthropodicus", an Intracellular Secondary Endosymbiont from the Hippoboscid Louse Fly Pseudolynchia canariensis. Society 72, 2997-3004. doi:10.1128/AEM.72.4.2997

Darling, A. E., Mau, B., and Perna, N. T. (2010). progressiveMauve: multiple genome alignment with gene gain, loss and rearrangement. PLoS One 5, e11147. doi:10.1371/journal.pone. 0011147

Drohojowska, J. and Szwedo, J. (2015). Early Cretaceous Aleyrodidae (Hemiptera: Sternorrhyncha) from the Lebanese amber. Cretac Res 52, 368-389. doi:10.1016/j.cretres.2014.03.015

Ellegaard, K. M., Klasson, L., Näslund, K., Bourtzis, K., and Andersson, S. G. E. (2013). Comparative genomics of Wolbachia and the bacterial species concept. PLoS Genet. 9, e1003381. doi:10.1371/journal.pgen.1003381

Ferrari, J. and Vavre, F. (2011). Bacterial symbionts in insects or the story of communities affecting communities. Philos. Trans. R. Soc. Lond., B, Biol. Sci. 366, 1389-1400. doi: $10.1098 /$ rstb.2010.0226

Gottlieb, Y., Ghanim, M., Gueguen, G., Kontsedalov, S., Vavre, F., Fleury, F., et al. (2008). Inherited intracellular ecosystem: symbiotic bacteria share bacteriocytes in whiteflies. The FASEB Journal 22, 2591-2599. doi:10.1096/fj.07-101162

Gottlieb, Y., Lalzar, I., and Klasson, L. (2015). Distinctive Genome Reduction Rates Revealed by Genomic Analyses of Two Coxiella-Like Endosymbionts in Ticks. Genome Biol Evol 7, 1779-1796. doi:10.1093/gbe/evv108

Guy, L., Kultima, J. R., and Andersson, S. G. E. (2010). genoPlotR: comparative gene and genome visualization in R. Bioinformatics 26, 2334-5. doi:10.1093/bioinformatics/btq413

Husnik, F. and McCutcheon, J. P. (2016). Repeated replacement of an intrabacterial symbiont in the tripartite nested mealybug symbiosis. Proc. Natl. Acad. Sci. U.S.A. 113, E5416-E5424. doi:10.1073/pnas.1603910113

Huson, D. H., Beier, S., Flade, I., Górska, A., El-Hadidi, M., Mitra, S., et al. (2016). MEGAN Community Edition - Interactive Exploration and Analysis of Large-Scale Microbiome Sequencing Data. PLoS Comput Biol 12, 1-12. doi:10.1371/journal.pcbi.1004957

Jones, P., Binns, D., Chang, H.-Y., Fraser, M., Li, W., McAnulla, C., et al. (2014). InterProScan 5: genome-scale protein function classification. Bioinformatics 30, 1236-1240. doi:10.1093/ bioinformatics/btu031

Kalyaanamoorthy, S., Minh, B. Q., Wong, T. K. F., von Haeseler, A., and Jermiin, L. S. (2017). ModelFinder: fast model selection for accurate phylogenetic estimates. Nat Methods 14, 587-589. doi:10.1038/nmeth.4285 
Kapantaidaki, D. E., Ovčarenko, I., Fytrou, N., Knott, K. E., Bourtzis, K., and Tsagkarakou, A. (2014). Low Levels of Mitochondrial DNA and Symbiont Diversity in the Worldwide Agricultural Pest, the Greenhouse Whitefly Trialeurodes vaporariorum (Hemiptera: Aleyrodidae). J Hered , 1-13doi:10.1093/jhered/esu061

Karp, P. D., Paley, S., and Romero, P. (2002). The Pathway Tools software. Bioinformatics 18 Suppl 1, S225-32

Katoh, K., Misawa, K., Kuma, K.-i., and Miyata, T. (2002). MAFFT: a novel method for rapid multiple sequence alignment based on fast Fourier transform. Nucleic Acids Res 30, 3059-66. doi:10.1093/nar/gkf436

Kearse, M., Moir, R., Wilson, A., Stones-Havas, S., Cheung, M., Sturrock, S., et al. (2012). Geneious Basic: An integrated and extendable desktop software platform for the organization and analysis of sequence data. Bioinformatics 28, 1647-1649. doi:10.1093/bioinformatics/ bts199

Kielbasa, S. M., Wan, R., Sato, K., Horton, P., and Frith, M. C. (2011). Adaptive seeds tame genomic sequence comparison. Genome Res 21, 487-493. doi:10.1101/gr.113985.110

Kirkness, E. F., Haas, B. J., Sun, W., Braig, H. R., Perotti, M. A., Clark, J. M., et al. (2010). Genome sequences of the human body louse and its primary endosymbiont provide insights into the permanent parasitic lifestyle. Proc. Natl. Acad. Sci. U.S.A. 107, 12168-73. doi:10.1073/pnas.1003379107

Konstantinidis, K. T. and Tiedje, J. M. (2005). Genomic insights that advance the species definition for prokaryotes. Proc. Natl. Acad. Sci. U.S.A. 102, 2567-72. doi:10.1073/pnas. 0409727102

Kurtz, S., Phillippy, A., Delcher, A. L., Smoot, M., Shumway, M., Antonescu, C., et al. (2004). Versatile and open software for comparing large genomes. Genome Biol 5, R12. doi:10.1186/gb-2004-5-2-r12

Lamelas, A., Gosalbes, M. J., Manzano-Marín, A., Peretó, J., Moya, A., and Latorre, A. (2011). Serratia symbiotica from the aphid Cinara cedri: a missing link from facultative to obligate insect endosymbiont. PLoS Genet 7, e1002357. doi:10.1371/journal.pgen.1002357

Langmead, B. and Salzberg, S. L. (2012). Fast gapped-read alignment with Bowtie 2. Nat Methods 9, 357-9. doi:10.1038/nmeth.1923

Latorre, A. and Manzano-Marín, A. (2016). Dissecting genome reduction and trait loss in insect endosymbionts. Ann N Y Acad Sci , 1-23doi:10.1111/nyas.13222

Li, L., Stoeckert, C. J., and Roos, D. S. (2003). OrthoMCL: identification of ortholog groups for eukaryotic genomes. Genome Res 13, 2178-89. doi:10.1101/gr.1224503

Luan, J.-B., Chen, W., Hasegawa, D. K., Simmons, A. M., Wintermantel, W. M., Ling, K.-S., et al. (2015). Metabolic Coevolution in the Bacterial Symbiosis of Whiteflies and Related Plant Sap-Feeding Insects. Genome Biol Evol 7, 2635-2647. doi:10.1093/gbe/evv170

Manzano-Marín, A. and Latorre, A. (2014). Settling Down: The Genome of Serratia symbiotica from the Aphid Cinara tujafilina Zooms in on the Process of Accommodation to a Cooperative Intracellular Life. Genome Biol Evol 6, 1683-98. doi:10.1093/gbe/evu133 
Manzano-Marín, A., Oceguera-Figueroa, A., Latorre, A., Jiménez-García, L. F., and Moya, A. (2015). Solving a Bloody Mess: B-Vitamin Independent Metabolic Convergence among Gammaproteobacterial Obligate Endosymbionts from Blood-Feeding Arthropods and the Leech Haementeria officinalis. Genome Biol Evol 7, 2871-2884. doi:10.1093/gbe/evv188

Manzano-Marín, A., Simon, J.-C., and Latorre, A. (2016). Reinventing the Wheel and Making It Round Again: Evolutionary Convergence in Buchnera - Serratia Symbiotic Consortia between the Distantly Related Lachninae Aphids Tuberolachnus salignus and Cinara cedri. Genome Biol Evol 8, 1440-1458. doi:10.1093/gbe/evw085

Manzano-Marín, A., Szabó, G., Simon, J.-C., Horn, M., and Latorre, A. (2017). Happens in the best of subfamilies: establishment and repeated replacements of co-obligate secondary endosymbionts within Lachninae aphids. Environ Microbiol 19, 393-408. doi:10.1111/ 1462-2920.13633

Mao, M., Yang, X., Poff, K., and Bennett, G. (2017). Comparative Genomics of the Dual-Obligate Symbionts from the Treehopper, Entylia carinata (Hemiptera: Membracidae), Provide Insight into the Origins and Evolution of an Ancient Symbiosis. Genome Biol Evol 9, 1803-1815. doi:10.1093/gbe/evx134

Marubayashi, J. M., Kliot, A., Yuki, V. A., Rezende, J. A. M., Krause-Sakate, R., Pavan, M. A., et al. (2014). Diversity and Localization of Bacterial Endosymbionts from Whitefly Species Collected in Brazil. PLoS One 9, e108363. doi:10.1371/journal.pone.0108363

Meseguer, A. S., Manzano-Marín, A., Coeur d'Acier, A., Clamens, A. L., Godefroid, M., and Jousselin, E. (2017). Buchnera has changed flatmate but the repeated replacement of coobligate symbionts is not associated with the ecological expansions of their aphid hosts. Mol Ecol 26, 2363-2378. doi:10.1111/mec.13910

Moriyama, M., Nikoh, N., Hosokawa, T., and Fukatsu, T. (2015). Riboflavin Provisioning Underlies Wolbachia's Fitness Contribution to Its Insect Host. MBio 6, e01732-15-e01732-15. doi:10.1128/mBio.01732-15

Moya, A., Peretó, J., Gil, R., and Latorre, A. (2008). Learning how to live together: genomic insights into prokaryote-animal symbioses. Nat Rev Genet 9, 218-29. doi:10.1038/nrg2319

Nakabachi, A. and Ishikawa, H. (1999). Provision of riboflavin to the host aphid, Acyrthosiphon pisum, by endosymbiotic bacteria, Buchnera. J Insect Physiol 45, 1-6. doi:10.1016/ S0022-1910(98)00104-8

Nawrocki, E. P. (2009). Structural RNA Homology Search and Alignment Using Covariance Models. Electronic theses and dissertations. paper 256., Washington University School of Medicine

Nguyen, L.-T., Schmidt, H. A., von Haeseler, A., and Minh, B. Q. (2015). IQ-TREE: A Fast and Effective Stochastic Algorithm for Estimating Maximum-Likelihood Phylogenies. Mol Biol Evol 32, 268-274. doi:10.1093/molbev/msu300

Nováková, E., Husník, F., Šochová, E., and Hypša, V. (2015). Arsenophonus and Sodalis symbionts in louse flies: An analogy to the Wigglesworthia and Sodalis system in tsetse flies. Appl Environ Microbiol 81, 6189-6199. doi:10.1128/AEM.01487-15 
Nováková, E., Hypsa, V., and Moran, N. a. (2009). Arsenophonus, an emerging clade of intracellular symbionts with a broad host distribution. BMC Microbiol 9, 143. doi:10.1186/ 1471-2180-9-143

Nováková, E., Hypša, V., Nguyen, P., Husník, F., and Darby, A. C. (2016). Genome sequence of Candidatus Arsenophonus lipopteni, the exclusive symbiont of a blood sucking fly Lipoptena cervi (Diptera: Hippoboscidae). Stand Genomic Sci 11, 72. doi:10.1186/s40793-016-0195-1

Nurk, S., Meleshko, D., Korobeynikov, A., and Pevzner, P. A. (2017). metaSPAdes: a new versatile metagenomic assembler. Genome Res 27, 824-834. doi:10.1101/gr.213959.116

[Dataset] Ouvrard, D. and Martin, J. H. (2018). The White-files - Taxonomic checklist of the world's whiteflies (Insecta: Hemiptera: Aleyrodidae).

Pirson, A. and Zimmermann, M. H. (1975). Transport in Plants I, vol. 1 (Berlin, Heidelberg: Springer Berlin Heidelberg). doi:10.1007/978-3-642-66161-7

Price, D. R. G. and Wilson, A. C. C. (2014). A substrate ambiguous enzyme facilitates genome reduction in an intracellular symbiont. BMC Biol 12, 110. doi:10.1186/s12915-014-0110-4

R Core Team (2018). R: A Language and Environment for Statistical Computing. R Foundation for Statistical Computing, Vienna, Austria

Rao, Q., Rollat-Farnier, P.-A., Zhu, D.-T., Santos-Garcia, D., Silva, F. J., Moya, A., et al. (2015). Genome reduction and potential metabolic complementation of the dual endosymbionts in the whitefly Bemisia tabaci. BMC Genomics 16, 226. doi:10.1186/s12864-015-1379-6

Rodriguez-R, L. M. and Konstantinidis, K. T. (2016). The enveomics collection : a toolbox for specialized analyses of microbial genomes and metagenomes. Peer J Preprints 4, e1900v1. doi:10.7287/peerj.preprints.1900v1

Russell, J. A., Oliver, K. M., and Hansen, A. K. (2017). Band-aids for Buchnera and B vitamins for all. Mol Ecol 26, 2199-2203. doi:10.1111/mec.14047

Santos-Garcia, D., Farnier, P.-A., Beitia, F., Zchori-Fein, E., Vavre, F., Mouton, L., et al. (2012). Complete genome sequence of "Candidatus Portiera aleyrodidarum" BT-QVLC, an obligate symbiont that supplies amino acids and carotenoids to Bemisia tabaci. J Bacteriol 194, 6654-5. doi:10.1128/JB.01793-12

Santos-Garcia, D., Latorre, A., Moya, A., Gibbs, G., Hartung, V., Dettner, K., et al. (2014a). Small but Powerful, the Primary Endosymbiont of Moss Bugs, Candidatus Evansia muelleri, Holds a Reduced Genome with Large Biosynthetic Capabilities. Genome Biol Evol 6, 18751893. doi:10.1093/gbe/evu149

Santos-Garcia, D., Rollat-Farnier, P.-A., Beitia, F., Zchori-Fein, E., Vavre, F., Mouton, L., et al. (2014b). The Genome of Cardinium cBtQ1 Provides Insights into Genome Reduction, Symbiont Motility, and Its Settlement in Bemisia tabaci. Genome Biol Evol 6, 1013-1030. doi:10.1093/gbe/evu077

Santos-Garcia, D., Silva, F. J., Morin, S., Dettner, K., and Kuechler, S. M. (2017). The allrounder Sodalis - a new bacteriome-associated endosymbiont of the lygaeoid bug Henestaris halophilus (Heteroptera: Henestarinae) and a critical examination of its evolution. Genome Biol Evol 9, 1-54. doi:10.1093/gbe/evx202 
Santos-Garcia, D., Vargas-Chavez, C., Moya, A., Latorre, A., and Silva, F. J. (2015). Genome Evolution in the Primary Endosymbiont of Whiteflies Sheds Light on Their Divergence. Genome Biol Evol 7, 873-888. doi:10.1093/gbe/evv038

Seemann, T. (2014). Prokka: rapid prokaryotic genome annotation. Bioinformatics 30, 20682069. doi:10.1093/bioinformatics/btu153

Segata, N., Börnigen, D., Morgan, X. C., and Huttenhower, C. (2013). PhyloPhlAn is a new method for improved phylogenetic and taxonomic placement of microbes. Nat Commun 4, 2304. doi:10.1038/ncomms3304

Shcherbakov, D. E. (2000). The most primitive whiteflies (Hemiptera; Aleyrodidae; Bernaeinae subfam. nov.) from the Mesozoic of Asia and Burmese amber, with an overview of Burmese amber hemipterans. Bull. nat. Hist. Mus. Lond. (Geol.) 56, 29-37

Skaljac, M., Zanic, K., Ban, S. G., Kontsedalov, S., and Ghanim, M. (2010). Co-infection and localization of secondary symbionts in two whitefly species. BMC Microbiol 10, 142. doi:10.1186/1471-2180-10-142

Skaljac, M., Zanić, K., Hrnčić, S., Radonjić, S., Perović, T., and Ghanim, M. (2013). Diversity and localization of bacterial symbionts in three whitefly species (Hemiptera: Aleyrodidae) from the east coast of the Adriatic Sea. Bull Entomol Res 103, 48-59. doi:10.1017/S0007485312000399

Slater, G. S. C. and Birney, E. (2005). Automated generation of heuristics for biological sequence comparison. BMC Bioinformatics 6, 1-11. doi:10.1186/1471-2105-6-31

Sloan, D. B. and Moran, N. A. (2012). Endosymbiotic bacteria as a source of carotenoids in whiteflies. Biol Lett 8, 986-989. doi:10.1098/rsbl.2012.0664

Šochová, E., Husník, F., Nováková, E., Halajian, A., and Hypša, V. (2017). Arsenophonus and Sodalis replacements shape evolution of symbiosis in louse flies. PeerJ 5, e4099. doi: $10.7717 /$ peerj.4099

Staden, R., Beal, K. F., and Bonfield, J. K. (2000). The Staden package, 1998. Methods Mol Biol 132, 115-30

Sudakaran, S., Kost, C., and Kaltenpoth, M. (2017). Symbiont Acquisition and Replacement as a Source of Ecological Innovation. Trends Microbiol 24, 932-976. doi:10.1016/j.tim.2017.02.014

Thao, M. L. and Baumann, P. (2004). Evidence for multiple acquisition of Arsenophonus by whitefly species (Sternorrhyncha: Aleyrodidae). Curr Microbiol 48, 140-4. doi:10.1007/ s00284-003-4157-7

Varani, A. M., Siguier, P., Gourbeyre, E., Charneau, V., and Chandler, M. (2011). ISsaga is an ensemble of web-based methods for high throughput identification and semi-automatic annotation of insertion sequences in prokaryotic genomes. Genome Biol. 12, R30. doi: $10.1186 / \mathrm{gb}-2011-12-3-\mathrm{r} 30$

Walker, B. J., Abeel, T., Shea, T., Priest, M., Abouelliel, A., Sakthikumar, S., et al. (2014). Pilon: An integrated tool for comprehensive microbial variant detection and genome assembly improvement. PLoS One 9. doi:10.1371/journal.pone.0112963

Wickham, H. (2009). ggplot2: Elegant Graphics for Data Analysis (Springer-Verlag New York) 
Wood, D. E. and Salzberg, S. L. (2014). Kraken: Ultrafast metagenomic sequence classification using exact alignments. Genome Biol 15. doi:10.1186/gb-2014-15-3-r46

Zchori-Fein, E., Lahav, T., and Freilich, S. (2014). Variations in the identity and complexity of endosymbiont combinations in whitefly hosts. Front Microbiol 5, 1-8. doi:10.3389/fmicb.2014. 00310 